\title{
Development of Composite Reverse Osmosis Membranes in Retrospect
}

\author{
John E. Cadotte \\ Film Tec Corporation; 7200 Ohms Lane, Minneapolis, MN 55435, U.S. A.
}

\begin{abstract}
Background
The Loeb-Sourirajan asymmetric membrane reported in 1960 was shown to consist of a porous matrix having a thin dense skin on one surface. This cellulose acetate membrane was used as a model to make a composite membrane at North Star Research Institute beginning in 1964. An ultrathin cellulose acetate membrane was formed on a water surface by the North Star float casting procedure. A microporous cellulose acetate film was formed separately and the two were laminated together. This approach allowed us to study separately the effects of variation in the properties of the microporous support and of the ultrathin membrane (also referred to as the "barrier layer"). The ultrathin membrane could be varied in thickness from 200 to 5000 angstroms.
\end{abstract}

From a practical point of view, these early cellulose acetate composite membranes proved to have no advantage over the asymmetric cellulose acetate membrane. However, one significant advance made in 1966 paved the way for improved composite membranes to follow. This was the development of microporous support films of engineering plastics such as polyphenylene oxide, polycarbonate, and particularly polysulfone. Polysulfone is still the preferred type in use today. As the limitations of the asymmetric cellulose acetate membranes became more apparent, we began to search for more durable polymers than cellulose esters to from the barrier layer. We tested many synthetic polymers for this purpose and, as expected, most failed because of inadequate water flux. One of the more interesting composite membranes developed during this period involved an acid-catalyzed condensation of furfuryl alcohol on a polysulfone support film. This membrane, called NS-200, exhibited salt rejections that were typically 99.9 percent in seawater tests. Unfortunately, this membrane did not possess long term stability, and it was not developed further at North Star Reseacharch Institute.

\section{Interfacially-Formed Membranes}

When a microporous support film is saturated with an aqueous diamine solution, then placed in contact with a waterimmiscible organic solvent containing a diacid chloride, a reaction at the interface produces a polyamide skin between the two phases. These interfacial reactions were reviewed in a book by P. W. Morgan in $1965^{*}$, and had been used to apply polyamide coatings to surfaces such as that of wool yarn. Morgan found that polyamide membranes, formed by interfacial reactions, were permeable to salt but retained organic dyes. With our background in the composite cellulose acetate membranes and having the polysulfone support film with good $\mathrm{pH}$ resistance, it was logical to attempt to make a polyamide barrier layer by the interfacial method. In the late 1960's many attempts were made to form interfacial membranes on polysulfone support films under conditions outlined by Morgan. The experiments utilized several types of diamines in the aqueous phase and

* Condensation Polymers by Interfacial and Solution Methods by P. W. Morgan, Interscience Publishers, 1965 . 
various diacid chlorides in the solvent phase. All of the membranes formed by these procedures appeared to be highly defective, and salt rejections were too low for practical seawater or brackish water desalination. Therefore, the conclusion at that time was that the interfacially formed membranes were inherently defective and efforts on their development were stopped.

In 1970 a new approach was tried for preparing the interfacial composite membranes. A polymeric polyamine was used in the aqueous phase rather than monomeric diamines. The polysulfone support film was saturated with the aqueous polyamine. After interfacial reaction with difunctional reagents such as diisocyanates or diacyl chlorides in hexane to form the barrier layer, the membrane was dried and heat-cured. The heat-cure crosslinked the polyamine layer on the support film to give a zone of intermediate porosity. This intermediate zone provided additional support for the very thin interfacially formed barrier layer. The membrane formed in this process was called NS-100. It was optimized in a relatively short time to give over 99 percent salt rejection in seawater reverse osmosis tests. Since that time many investigators have synthesized and used a wide variety of polyamines in place of polythylenimine in this type of membrane.

\section{Monomeric Amine Reactants.}

For a period of several years it was thought that the polyamine reactant was essential to give membranes retentive enough for seawater use. In 1975 we were engaged in a research program to prepare chlorine-resistant composite membranes. One approach in the program was to use piperazine-containing polyamides for which some of the technical literature claimed greater resistance to oxidation by chlorine. Optimization of the interfacial reaction conditions between piperazine and isophthaloyl chloride produced composite membranes with low flux, but having salt rejections in the upper 90 percent range in seawater tests.

Although the piperazineamide interfacial membranes produced in this work were not as chlorine-resistant as expected, their relatively improved performance encouraged us to continue research on interfacial reactions using monomeric diamine reactants. This effort culminated in 1978 with the development of the FT-30 membrane made from aromatic diamines and triacyl chloride reactants. The FT-30 membrane had high flux and it rejected over 99 percent of the salt in seawater tests. Studies of the structure of FT-30 membrane with the scanning electron microscope showed rough appearance on the face of the membrane. The back surface of a small area of the barrier layer, peeled away from the polysulfone support, showed numerous pinholes. We interpreted these SEM observations to reflect on the mechanism of membrane formation. It appears that first contact between the aqueous diamine phase with the acid chloride organic phase in the FT-30 reaction produced a defective membrane with pinholes. However, unlike the numerous other diamine-acid chloride reactions investigated in the past, the reaction continued to close up essentially all the defects by building more membrane over the top of the pinholes.

In conclusion, a suitable support film and the knowledge of the chemistry of interfacial reactions were available to us in the late 1960's. We were not able to apply these concepts immediately in the preparation of effective composite membranes, because the rapid interfacial reactions tended to produce pinholes. Two methods were eventually developed to produce high quality membrane: 1) the use of polymeric amines as in the NS-100 and 2) in the case of FT-30 the selection of reactants and concentrations that allow control of barrier layer formation. 